\title{
Autonomous Operations Planner: A Flexible Platform for Research in Flight-Deck Support for Airborne Self- Separation
}

\author{
David A. Karr, ${ }^{*}$ Robert A. Vivona, ${ }^{\dagger}$ David A. Roscoe,${ }^{\ddagger}$ Stephen M. DePascale ${ }^{\S}$ \\ Engility Corporation, Billerica, MA 01821, USA \\ and \\ David J. Wing ${ }^{* *}$ \\ NASA Langley Research Center, Hampton, VA 23681, USA
}

\begin{abstract}
The Autonomous Operations Planner (AOP), developed by NASA, is a flexible and powerful prototype of a flight-deck automation system to support self-separation of aircraft. The AOP incorporates a variety of algorithms to detect and resolve conflicts between the trajectories of its own aircraft and traffic aircraft while meeting route constraints such as required times of arrival and avoiding airspace hazards such as convective weather and restricted airspace. This integrated suite of algorithms provides flight crew support for strategic and tactical conflict resolutions and conflict-free trajectory planning while en route. The AOP has supported an extensive set of experiments covering various conditions and variations on the self-separation concept, yielding insight into the system's design and resolving various challenges encountered in the exploration of the concept. The design of the AOP will enable it to continue to evolve and support experimentation as the self-separation concept is refined.
\end{abstract}

\section{Nomenclature}

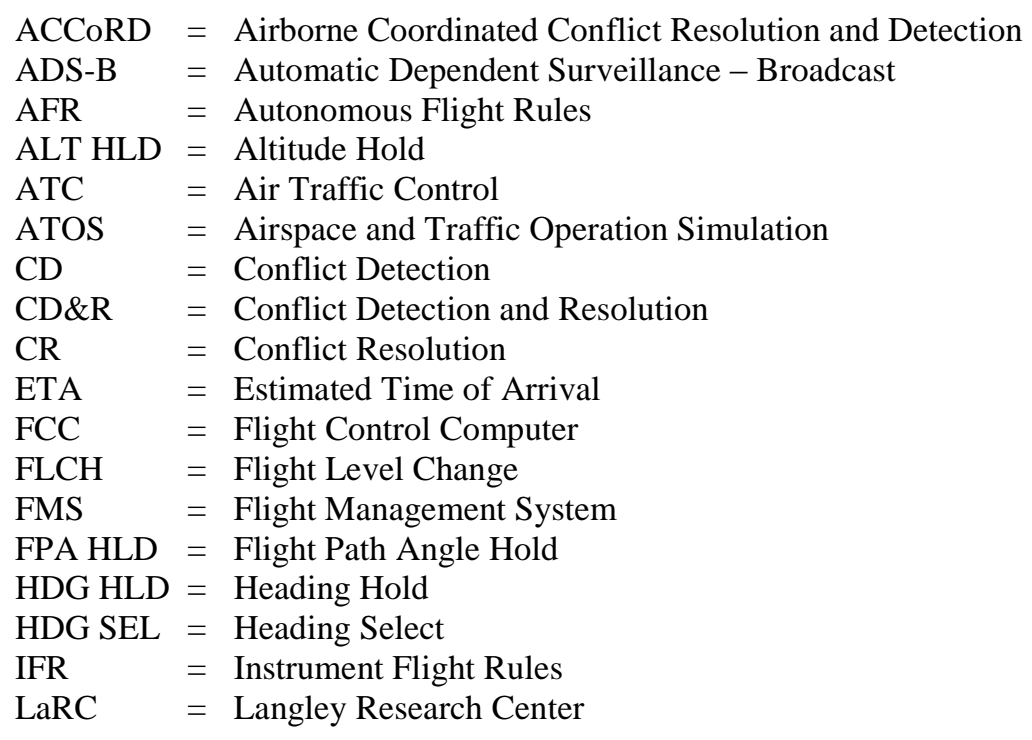

* Principal Computer Scientist, ATRE Department, 300 Concord Road, AIAA member. 978-671-1110.

${ }^{\dagger}$ Chief Research Engineer, ATRE Department, 300 Concord Road, AIAA associate fellow. 978-671-1116.

\$ Senior Software Engineer, ATRE Department, 300 Concord Road, AIAA member. 978-671-1027.

${ }^{\S}$ Senior Software Engineer, ATRE Department, 300 Concord Road, AIAA senior member. 978-671-1044.

** ATM Research Engineer, Crew Systems \& Aviation Operations, M.S. 152, AIAA member. 757-864-3006 


$\begin{array}{ll}\text { LNAV } & =\text { Lateral Navigation } \\ \text { LOS } & =\text { Loss of Separation } \\ \text { MCDU } & =\text { Multifunction Control Display Unit } \\ \text { MCP } & =\text { Mode Control Panel } \\ \text { MR } & =\text { Maneuver Restriction } \\ \text { PBGA } & =\text { Pattern-Based Genetic Algorithm } \\ \text { RTA } & =\text { Required Time of Arrival } \\ \text { SICR } & =\text { Strategic Intent-based Conflict Resolution } \\ \text { SNAPI } & =\text { Status Notifications and Planning Indicators } \\ \text { SVR } & =\text { State Vector Report } \\ \text { SWIM } & =\text { System Wide Information Management } \\ \text { TCP } & =\text { Trajectory Change Point } \\ \text { TCR } & =\text { Trajectory Change Report } \\ \text { TICR } & =\text { Tactical Intent-based Conflict Resolution } \\ \text { TPUBs } & =\text { Trajectory Prediction Uncertainty Bounds } \\ \text { TRK HLD } & =\text { Track Hold } \\ \text { TRK SEL } & =\text { Track Select } \\ \text { TSR } & =\text { Target State Report } \\ \text { V/R HLD } & =\text { Vertical Rate Hold } \\ \text { VNAV } & =\text { Vertical Navigation }\end{array}$

\title{
I. Introduction
}

\begin{abstract}
A s commercial airspace continues to suffer from congestion, heavy workloads on current air traffic control (ATC) personnel and systems, unpredictable local weather conditions, and other effects of airspace complexity, research continues on ways to alleviate these problems.

One approach that has been investigated is to equip aircraft to perform self-separation, a procedure by which an aircraft provides for its own separation from other aircraft. A flight-deck system designed for this purpose can perform several functions. For example, such a system can alert an air crew to a potential loss of separation with other aircraft or penetration of severe weather conditions. The system can also probe alternative trajectories of the aircraft in order to avoid these problems. This can enable crews to self-separate from other aircraft, as proposed under the Autonomous Flight Rules (AFR) concept. ${ }^{1}$ Alternatively, under conventional Instrument Flight Rules (IFR), crews might use such a system as an aid to composing requests to ATC for clearances that are more likely to be granted.

The Autonomous Operations Planner (AOP), developed under contract to NASA Langley Research Center, is a prototype of such a system. Designed as a research tool, it is an instrument for investigating the properties and benefits of a variety of systems of this general type that might be implemented. The AOP has been extensively studied within the Airspace and Traffic Operation Simulation (ATOS) platform, a networked flight-deck simulation environment that models real-world standard interfaces and enables extensive feasibility testing of ATM concepts. ${ }^{2}$

In the remaining sections of this paper, we discuss the motivation for flight-deck support for airborne selfseparation. We document several functions that can be performed by the AOP in the context of research into air traffic management concepts. We show some issues in the general design of such systems that have been illustrated during the development and experimental usage of the AOP. Finally, we comment on the future directions this development and experimentation may take.
\end{abstract}

\section{Motivations for Flight-Deck Support for Airborne Self-Separation}

The concept of autonomous flight with self-separation has been studied since at least $1965 .{ }^{3}$ By performing some of the functions of separation of air traffic, a system on the aircraft flight deck can help reduce the load on groundbased systems, especially as airspace becomes more complex. ${ }^{4}$

The AFR concept, in particular, promises more complete use of airspace between the jet routes, potentially raising capacity or mitigating capacity loss due to weather systems while providing substantial user benefits in flight efficiency and operational flexibility. ${ }^{5}$

Automated support for airborne self-separation is necessary even for relatively simple tasks, largely because it is difficult for pilots to visualize the interactions between aircraft. This difficulty is exacerbated when aircraft are turning, climbing, or descending, or have an intent to turn, climb, or descend later. The difficulty further increases as the number of proximate aircraft increases, and increases again in the presence of weather and special-use airspaces 
(SUAs) that aircraft must avoid. A maneuver to avoid one hazard (such as traffic or weather) may put the maneuvering aircraft in the way of a different hazard. Finally, the aircraft may also be subject to arrival flow management constraints such as a required time of arrival (RTA) at a fixed waypoint, which must be met by any proposed maneuver. Controllers have need for automated support to avoid problems, air crews even more so.

The AOP seeks to support airborne self-separation at many levels of complexity. Its intended applications range from homogeneous operations to mixed air-ground separation operations; from current-day traffic density to many multiples of current day; and from highly flexible, unconstrained operations to flight constrained by arrival times, airspace hazards, and aircraft performance limits. Figure 1 is a simplified illustration of this concept.

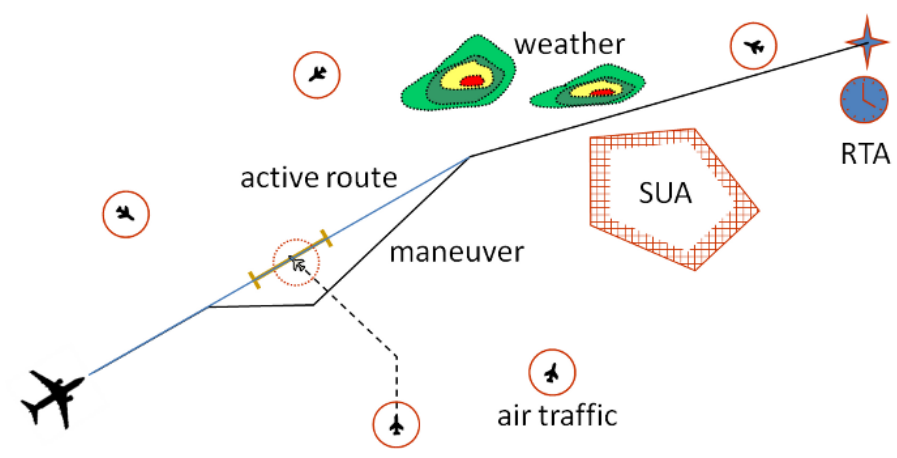

Figure 1. Global trajectory based self-separation with constraints.

\section{Basic Concepts and Definitions to Support Self-Separation}

The explanation of the capabilities of the AOP will rely on the following definitions:

\section{A. The Ownship}

Because of the nature of a flight-deck system, most of the phenomena involving the AOP are most easily described from the perspective of a single AOP-equipped aircraft. Except when otherwise indicated, therefore, the following definitions and discussion refer to one such aircraft as the ownship aircraft (or simply "the ownship"), and "the AOP" refers to the instance of the AOP that is installed and operating on the ownship.

\section{B. Hazards}

There are two main classes of hazards defined in AOP:

- All aircraft in flight other than the ownship are considered traffic aircraft. Traffic aircraft constitute one main class of hazards.

- The other main class of hazards, area hazards, consists of weather cells, SUAs, terrain, and any other such three-dimensional regions that are excluded from the usable airspace.

Due to practical considerations, a system such as the AOP should not assume that area hazards are permanent or fixed in space and time. Some SUAs apply only during predetermined time intervals; weather cells can appear, disappear, or move in a relatively unpredictable fashion.

\section{Aircraft States and Intents}

The AOP considers a state of an aircraft to consist of instantaneous data, such as the aircraft's pressure altitude, latitude, longitude, airspeed, groundspeed, heading, true course or track angle, vertical rate, and aircraft weight, that can be sensed or derived from sensed data. An intent of an aircraft consists of any information that AOP might receive about how the aircraft's guidance systems will control its flight path. From a given state and intent, it is possible to predict or infer future states of the aircraft. Any change to that intent that results in different predicted future states is considered to be a maneuver.

\section{Conflicts}

Consistent with the precedent of today's ground-based separation services for aircraft under IFR, each aircraft has a certain required lateral separation and a certain required vertical separation. For example, a typical set of separation requirements considered in experiments with the AOP has been five miles lateral separation for AOPequipped aircraft (reflecting existing separation requirements), with an additional buffer around ground-separated IFR aircraft (providing additional assurance that AOP-based operations do not interfere with conventional operations), and 1000 feet vertical separation for all aircraft. A loss of separation (LOS) between the ownship and a traffic aircraft occurs when two conditions apply simultaneously: 
- the lateral distance between the two aircraft is less than the defined minimum separation standard between these aircraft, and

- the magnitude of the difference between the altitudes of the aircraft is also less than the prescribed minimum separation.

We also say that LOS occurs between the ownship and an area hazard when the ownship penetrates that area hazard, that is, when the ownship is within the region defined by that hazard at that time.

A conflict is a predicted LOS, that is, a LOS that might occur based on predicted future states of the ownship and hazards. A conflict may be either:

- a traffic conflict, consisting of predicted states of the ownship and a traffic aircraft that will be in LOS at the time when those states are predicted to occur; or

- an area-hazard conflict, consisting of an area hazard and a predicted state of the ownship that will penetrate the region occupied by that hazard at the time when that state is predicted to occur.

\section{E. Conflict Resolution and Conflict Prevention}

An overarching objective of AOP is to perform conflict resolution (CR), that is, to detect conflicts and resolve them by recommending maneuvers that will avoid LOS. In addition to this primary flight-safety function, AOP also has the objective of conflict prevention, the process of planning maneuvers that do not cause conflicts between the ownship and other aircraft. Integrated conflict resolution and prevention in AOP provides maneuvers that are free of all conflicts while enabling pilots to avoid conflicts in other contemplated maneuvers.

\section{F. Intent-Based, State-Based, Strategic, and Tactical Functions}

The AOP considers the ownship to be in a strategic flight mode when it is following the active route of the Flight Management System (FMS) with the Lateral Navigation (LNAV) and Vertical Navigation (VNAV) functions of the FMS engaged. When the ownship is not under LNAV and VNAV guidance, it is considered to be in a tactical flight mode. Various "strategic" and "tactical" functions are related to the strategic and tactial flight modes, respectively.

Most of the functions of the AOP are intent-based, that is, they attempt to account for the intent of an aircraft to the extent that the intent is specified. Some intent-based functions are denoted as "strategic" or "tactical" functions depending on the flight modes of the intents those functions might receive. State-based functions, on the other hand, never use any information about aircraft intent, and are always considered tactical.

For ease of exposition, the next several sections of the paper describe intent-based functions before considering state-based functions and their relationship to the intent-based functions.

\section{Intent-Based Trajectory Prediction}

Clearly, the usefulness of either conflict resolution or conflict prevention depends on making appropriate predictions of conflicts, which in turn depends on predictions of the ownship trajectory as well as traffic trajectories and area-hazard locations. For the purpose of intent-based functions, the AOP predicts trajectories as described below.

\section{A. Intent-Based Ownship Trajectory Prediction}

1. Supported Flight Modes

The AOP can predict intent-based ownship trajectory based on the ownship's state and either the ownship's actual intent or a hypothetical intent. The AOP can predict trajectories for intents in various flight modes as determined by data that have been received (or that could be received) from the the Flight Control Computer (FCC), the Mode Control Panel (MCP), and optionally the FMS. Lateral modes include LNAV, track hold, track select (TRK SEL), heading hold, and heading select (HDG SEL), where tracks and headings may be relative to magnetic north or true north. Vertical modes include VNAV ${ }^{\dagger \dagger}$ altitude hold (ALT HLD), ${ }^{\dagger}$ vertical rate hold (V/R HLD), flight path angle hold (FPA HLD), and flight level change (FLCH).

Ideally, when the intent specifies "pure" LNAV and VNAV guidance, the AOP's prediction will be identical to that of the FMS. (In fact, the AOP uses the prediction code from the prototype FMS in its research environment

\footnotetext{
"Unless otherwise specified, "VNAV" denotes VNAV PATH or VNAV SPD, whichever is appropriate to follow the speed and altitude profile of the relevant FMS route. The AOP also recognizes a VNAV ALT mode; this mode behaves like altitude hold, and is not discussed further in this paper.

"A "hold" mode signifies that the named property is maintained at its current value, that is, constant.
} 
when it predicts these trajectories.) If the intent specifies LNAV and VNAV guidance but also specifies a commanded altitude setting of the MCP, the initial portion of the predicted trajectory will be identical to the trajectory of the "pure" LNAV/VNAV intent, but will "level off" at the commanded altitude at the first point where the trajectory would otherwise cross or depart from that altitude. Trajectories for tactical intents (namely, all other combinations of FCC and MCP settings) are predicted by a modular design that allows the trajectory prediction code to combine the equations of motion for appropriate lateral and vertical profiles over any time interval of the trajectory, and to detect the points along the trajectory at which those equations should change.

2. Trajectory Data Structure and Lookahead Time

The predicted trajectory of the ownship consists primarily of a sequence of discrete states, each of which is considered a multi-dimensional "point" along the trajectory. A principal goal of trajectory prediction is to assign a latitude, longitude, altitude, and time at each of these trajectory points as accurately as possible, as these are the properties that support conflict detection and related functions. On the other hand, in order to support these functions, it is generally sufficient for the AOP to generate trajectory points that span a time interval of a predetermined length, starting at the current time. (The length of this interval is called the lookahead time of the trajectory.) The AOP therefore seldom generates a sequence of trajectory points all the way to the end of a flight. The predicted trajectory may, however, include additional information (such as aircraft weight at destination) computed for events later than the last trajectory point, In particular, if the ownship intent is to follow full LNAV and VNAV guidance to a waypoint with a time constraint, the AOP will compute a predicted time of arrival at that waypoint and will record the difference between that predicted time and the RTA at that waypoint.

\section{B. Intent-Based Traffic Trajectory Prediction}

Traffic trajectories are based on data received by the ownship from external data sources. Past research using the AOP has relied almost exclusively on Automatic Dependent Surveillance - Broadcast (ADS-B) reports sent by the traffic aircraft for both states and intents of traffic aircraft. An intent-based traffic trajectory may be inferred from any of the following combinations of ADS-B reports:

- a State Vector Report (SVR) and either a Trajectory Change Report (TCR) or a cycle of multiple TCRs;

- $\quad$ an SVR and a Target State Report (TSR); or

- an SVR alone.

When the traffic aircraft provides TCRs, each TCR specifying a trajectory change point (TCP) with a latitude, longitude, altitude, and estimated time of arrival (ETA), ${ }^{\S \S}$ the mean ground speed and mean vertical rate between two TCPs can be estimated from the path length, difference in altitude, and difference in ETA along the portion of the trajectory between the two TCPs. Since the source of these data is the transmitting aircraft's navigation system, this trajectory represents a high-quality prediction of the aircraft's future intent.

Whereas some aircraft may broadcast TCRs, others may not. If a traffic aircraft sends a TSR but no TCR, the target state (from the TSR) enables the AOP to model an initial turn or a level-off point at a specified altitude in the predicted trajectory. This typically results in a lower-quality prediction than can be obtained with a cycle of TCRs.

If the traffic aircraft sends neither a TCR nor a TSR, the AOP will predict a trajectory by means of a state projection forward from the most recent SVR. In other words, the AOP will assume that the traffic aircraft starts at the latitude, longitude, altitude, and time reported in the SVR, and assumes that the aircraft flies at a constant ground speed and vertical speed along the great-circle course corresponding to its initial position and ground track. While this is not obviously an "intent-based" trajectory, it is treated as one for the purpose of all intent-based functions, since it represents the AOP's best effort to use any available intent data in the trajectory prediction.

The use of data sources other than ADS-B is limited only by the requirement for the existence of software functions to infer a sequence of predicted states from the incoming data. Recently, Flight Object data from the System Wide Information Management (SWIM) program have been considered as a basis for inferring traffic trajectories in the AOP. ${ }^{* * *}$ The latitudes, longitudes, altitudes, and ETAs at consecutive points in a Flight Object uplinked to an AOP-equipped aircraft can be used to estimate mean ground speed and mean vertical rate along the path between the two points. When SWIM and ADS-B are both available for a particular traffic aircraft, a proposed method for trajectory prediction is to prefer to predict a trajectory from TCRs (if available) rather that SWIM, but to prefer to use SWIM (if available) rather than a TSR.

${ }_{\S}^{\S}$ ETA is not explicitly specified by a TCR, but can be computed by adding the time to go (TTG) to the time of applicability (TOA).

In the AFR concept, the provision of Flight Object data to aircraft as part of SWIM is a conceptual alternative to widespread ADS-B intent broadcast. 


\section{Intent-Based Conflict Detection}

Conflict detection (CD) is the process of finding conflicts between the ownship and hazards. Intent-based CD is $\mathrm{CD}$ that uses intent-based trajectories. The AOP can perform a probe of any intent-based ownship trajectory by comparing states predicted by the selected ownship trajectory to states predicted by the trajectory of each traffic aircraft and to the predicted locations of each area hazard known to the AOP. This probe may detect one or more conflicts with traffic aircraft or area hazards.

When a conflict occurs, there is a range of predicted ownship states spanning a period of time (usually longer than a single instant) during which each ownship state is in LOS with the hazard that caused this conflict. The earliest predicted ownship state in this range is called the first $L O S$ with respect to this conflict.

It is readily apparent that the usefulness of the AOP-namely, its ability to effectively resolve or prevent conflicts - depends in large part on the accuracy of CD, that is, the ability to predict whether a given ownship intent will result in LOS with one or more hazards. Experience has supported that statement. The accuracy of CD, in turn, clearly depends on the ability to accurately predict ownship states, traffic states, and the applicable times and locations of area hazards.

\section{A. Causes and Effects of Uncertainty in Predictions}

For several reasons, it is generally not practical to predict aircraft trajectories perfectly. The path of an aircraft is influenced by many interacting factors, including guidance algorithms, control surfaces, throttle settings, properties of the airframe, and properties of the surrounding air mass (including temperature, density, and the speed and direction of wind). In the course of any prediction over a time interval that is useful to the AOP, some of these factors (such as the future wind fields into which the aircraft will fly) are subject to unpredictable variations that can cause significant prediction errors, that is, differences between predicted and actual aircraft states. There is therefore an unavoidable prediction uncertainty inherent in any predicted trajectory.

Traffic trajectories suffer from additional sources of prediction error. A cycle of TCRs will not describe all the details (such as variations in speed or flight path angle during a descent) that can be modeled in a trajectory produced by the AOP or by an FMS. Moreover, an aircraft may not send TCRs, and TCRs sent may not be received, forcing a fallback to even less accurate modeling of traffic trajectories.

In the presence of these sources of uncertainty, the AOP makes certain other approximations in its predictions for the sake of computational feasibility. For example, the AOP may have to compute ownship paths covering several orders of magnitude more cycles of the guidance systems than the aircraft actually flies in the same period of time. ${ }^{6}$ The interactions among guidance, airframe, engines, and the atmosphere are simplified, and the points along a trajectory predicted by AOP represent a piecewise linear approximation of non-linear motion. The combined effect of these approximations is expected to be small in comparison to other sources of error that cannot be avoided.

If trajectory predictions, area-hazard locations, and the conflict detection algorithm were all completely accurate, detection of a conflict would indicate that a LOS would occur if no change is made to the ownship intent or (if this is a traffic conflict) the traffic aircraft intent. In fact, prediction errors can result in false positive conflicts ("false alerts" in which a conflict is detected but no LOS actually would have occurred) and false negative conflicts ("missed alerts" in which the aircraft would actually fly into LOS but no conflict is detected).

\section{B. Accounting for Uncertainty}

An obvious way to reduce prediction uncertainty is to address the sources of error in predictions. Once this process has been carried to a certain point, however, the benefit of further accuracy improvements will be outweighed by their costs, especially if the remaining uncertainty is dominated by unavoidable components. After that point, the approach to $\mathrm{CD}$ is typically driven by the perception that the cost of a missed alert (in terms of safety and eventual crew workload) is greater than the cost of a false alert. One therefore implements techniques that are expected to reduce the frequency of missed alerts while accepting the idea that these techniques will likely increase the frequency of false alerts.

One way to reduce the frequency of missed alerts is to add "buffers" to the lateral and vertical separation requirements when performing conflict detection. For example, if $\mathrm{CD}$ assumes that 5.2 nautical miles lateral separation is required, a "no conflict" result implies a LOS could occur only at a time when the lateral positional error of the ownship (or combined lateral positional error of ownship and traffic aircraft) is at least 0.2 nautical miles. For this reason (among others), the separation requirements in the AOP can be independently configured for different research experiments.

A somewhat more sophisticated approach is to make the separation requirements between aircraft a function of time or of distance traveled. This approach neglects the observation that in typical operations, the positional error of 
aircraft trajectories along their tracks at any given time has a very different probability distribution than the error in the perpendicular (cross-track) lateral direction. It is therefore advantageous to introduce a directional bias in the algorithm, for example by modeling an ellipsoidal probability distribution of the aircraft's position relative to the nominal trajectory (which is the simple path described earlier in the context of trajectory prediction). ${ }^{7}$

The approach taken in the AOP is to model three independently-measured dimensions of prediction error around the nominal trajectory. For each consecutive pair of points of the nominal trajectory that the AOP stores in its prediction, the AOP computes trajectory prediction uncertainty bounds (TPUBs) that define a four-dimensional "box" around the segment of the trajectory between those two points. In order to relate prediction errors independently to different segments of the trajectory, the AOP models along-path errors as follows:

- An error that results in the aircraft being farther along the path at a given time than the nominal prediction for that time is treated as arriving "too early" at the point actually reached.

- An error that results in the aircraft being less far along the path at a given time than the nominal prediction for that time is treated as arriving "too late" at the point actually reached.

Along-path error is therefore expressed in terms of a "time of arrival error" at a given distance along the path.

The TPUBs can independently specify altitude bounds above the nominal path, altitude bounds below the nominal path, cross-track bounds, and along-path bounds. Moreover, the "box" can be "tapered" by allowing any of the independent bounds to increase or decrease linearly from one trajectory point to the next. (The bounds along the segment between any pair of points are therefore controlled by eight mutually-independent parameters.) The CD algorithm then finds conflicts that can result from any state inside those bounds. ${ }^{8}$

Since these TPUBs are assigned during the procedure of trajectory prediction, when the flight modes that generate the trajectory are known, the TPUBs can be made to fit a unique model of uncertainty for each flight mode, and even to fit a unique model of uncertainty for each segment or phase of a flight mode. Because of this feature, and because the model of along-path error uses distance along path rather than time as its independent dimension, the AOP can construct a continuous, self-consistent set of TPUBs that follows any trajectory around turns, across a transition into or out of a climb or descent, or through any other change in speed, vertical rate, or flight path angle. Moreover, the TPUBs can be tuned to the predicted behavior of the aircraft at any point along its trajectory in such a way as to prevent the class of missed alerts anticipated at that point while accepting a near-minimal increase in the likelihood of a false alert at that point.

\section{Closest Point of Approach}

During the procedure of conflict detection, the AOP monitors the closest point of approach of each traffic aircraft relative to the ownship. This information is made available to cockpit displays for use in "filtering" the traffic aircraft that are to be displayed to the crew.

\section{Active Trajectory Probing}

The AOP is able to notify the air crew when the current intent of the ownship is likely to cause problems, including conflicts with traffic aircraft, conflicts with area hazards, or missing an RTA at a waypoint ahead of the aircraft on the active route of the FMS.

In order to support this capability, the AOP periodically performs conflict probes-a primary probe and sometimes a secondary probe - according to the procedures described below.

\section{A. The Primary Probe}

The primary probe is performed on an ownship trajectory that is based on an intent derived from the current settings of the FCC, MCP, and FMS. When the vertical guidance mode is ALT HLD, V/R HLD, FPA HLD, or $\mathrm{FLCH}$, the trajectory is predicted using all of these settings, assuming that the crew make no modifications to the intent at any time. In the VNAV guidance mode, however, it is assumed that the trajectory of primary interest is the one that follows the vertical profile determined by VNAV, without regard to the commanded altitude currently set on the MCP. That is, the primary probe uses a trajectory that does not "level off" as described in Section IV. For the purpose of this probe, it is assumed that the crew will adjust the commanded altitude on the MCP as needed to prevent level-off.

The interpretation of commanded altitude in the primary probe is based on the experience of professional pilots using the AOP in studies of the AFR concept. For example, when the ownship is in the cruise segment of an FMS active route with LNAV and VNAV guidance engaged, a typical procedure pilots might follow is to leave the commanded altitude set at the cruise altitude until shortly before the aircraft needs to descend. If the ownship is cruising at 30,000 feet, and the next waypoint in the route is constrained to a maximum altitude of 10,000 feet, the 

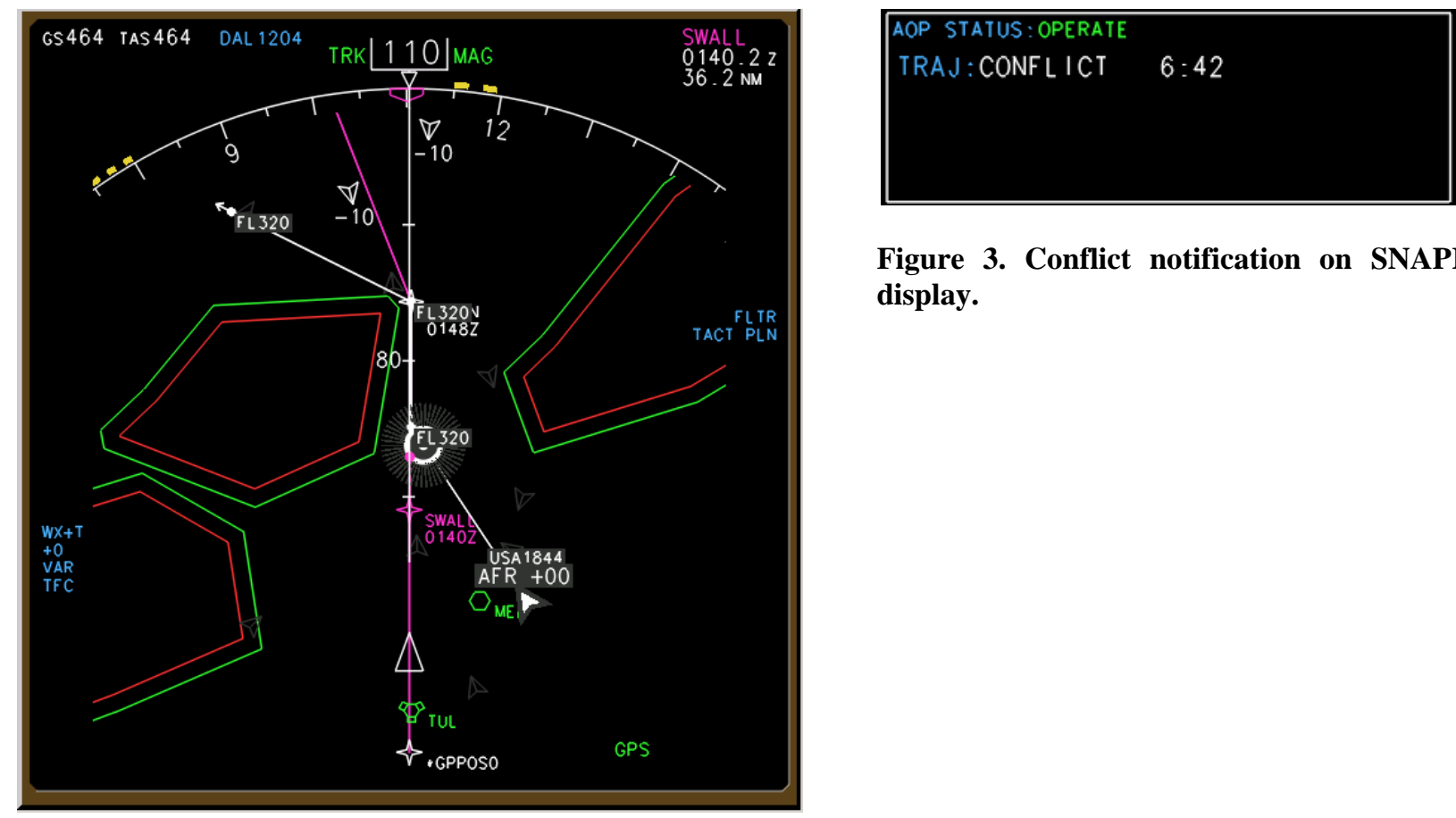

Figure 3. Conflict notification on SNAPI display.

Figure 2. Conflict notification on the ND.

FMS will have plotted the point at which the top of descent (TOD) should occur for the descent to that waypoint. In that case, the crew typically will adjust the commanded altitude to 10,000 feet shortly before the ownship reaches that TOD.

Any conflict detected by this probe is a primary conflict.

\section{B. The Secondary Probe}

The secondary probe is performed only when all of the following conditions are true:

- the VNAV guidance mode is engaged, and

- the current setting of the commanded altitude eventually would cause the ownship to level off, and

- the interval from the current time to the level-off point is less than the lookahead time of the trajectory used in the primary probe.

When the secondary probe is performed, it uses a trajectory that is predicted from the exact intent determined by the current settings of the FCC, MCP, and FMS, including the commanded altitude. This trajectory "levels off" at the point where the VNAV vertical profile would have crossed or departed from the commanded altitude.

The secondary probe is performed because there is an ambiguity in the translation of the aircraft's current guidance modes to the intent from which the primary probe's trajectory is predicted. The crew may have a reason not to adjust the commanded altitude before flying past the TOD computed by the FMS. In that case, the aircraft might fly into a LOS that the primary probe cannot predict, because the level-off has caused the ownship to depart from the trajectory predicted by the primary probe. The secondary probe is intended to detect any conflict corresponding to such a LOS. Such a conflict is a secondary conflict.

\section{Alerting the Crew to Results of Probes}

The AOP has implemented several techniques to alert the crew when a conflict occurs in either a primary probe or a secondary probe. Figure 2 illustrates the notification of a primary conflict on the navigation display (ND), and Fig. 3 shows the corresponding notification on the Status Notifications and Planning Indicators (SNAPI) display.

When the ownship's LNAV and VNAV guidance modes are engaged and the active route includes an RTA, the AOP also notifies the crew if the ownship will "miss" the RTA, that is, if the ownship is predicted to arrive too soon or too late at that RTA. 


\section{Conflict Prevention}

Unlike Active Conflict Detection capabilities that detect conflicts with the aircraft's current guidance settings, AOP's Conflict Prevention capabilities detect conflicts for proposed changes to the current settings. AOP has four separate conflict prevention capabilities: FMS MOD Route probe, Tactical Planning probe, Active Route Recouple probe, and Maneuver Restriction (MR) bands. Each of these capabilities analyzes one or more potential changes to the aircraft's current guidance settings and alerts the flight crew to any predicted conflicts prior to implementing the guidance changes. Conflict prevention is a critical component of the AFR concept because these capabilities enable the flight crew to modify their flight path at their discretion (i.e., without direction from AOP) while avoiding the creation of unacceptable conflicts with other aircraft. It is these capabilities that enable the flight crew to have flexibility in choosing their desired guidance approach while maintaining the requirements of self-separation.

The three conflict prevention probes (FMS MOD Route, Tactical Planning, and Active Route Recouple) each have their own automatic triggering mechanism and their own indicator line on the SNAPI (see Fig. 4). The triggering and display options are described in the capability descriptions below. In contrast, MR Bands are continuously calculated and displayed on the Primary Flight Display (PFD) and Navigation Display.

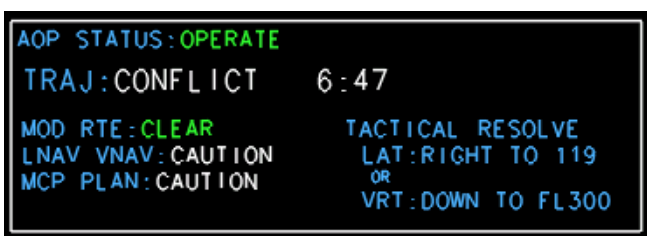

Figure 4. SNAPI representation for conflict prevention probes.

\section{A. FMS MOD Route Probe}

The FMS MOD Route probe is automatically initiated by AOP whenever a MOD route is created in the FMS. The purposes of the FMS MOD Route probe are to alert the crew to any undesirable impacts on AFR procedures if the current MOD route were implemented and to enable these impacts to be resolved prior to implementation.

A MOD route represents a proposed modification to the FMS's active route and is implemented in the guidance system by executing the MOD route and engaging LNAV and VNAV. When executed, the MOD route becomes the new active route by replacing the previous active route. MOD routes can be generated by manual flight crew inputs in the Multifunction Control Display Unit (MCDU), an uplinked route via datalink, or an upload of a route modification from AOP. The FMS displays MOD route information on the MCDU and ND so that the flight crew can evaluate any impacts on navigation and guidance prior to implementation. AOP's FMS MOD Route probe complements this information by providing any impacts on the aircraft's self-separation requirements.

AOP's FMS MOD Route probe has two main functions:

- Perform conflict detection on the MOD route to identify what conflicts would exist if the MOD route were executed with LNAV and VNAV engaged.

- Determine if the MOD route would fail to achieve any required time of arrival (RTA) constraints that exist in the route if the MOD route were executed with LNAV and VNAV engaged.

If the MOD route is conflict-free and meets all of its RTA constraints, the SNAPI will indicate "CLEAR" on the line entitled MOD RTE, alerting the flight crew that it is safe to execute the MOD route and engage LNAV and VNAV (if not already engaged). If a conflict is detected along the MOD route, the SNAPI will indicate "CAUTION" or "NOT AVBL" (not available), alerting the flight crew that a conflict will be created if the MOD route is executed. If the MOD route is conflict-free but unable to achieve an RTA constraint defined in the route, the SNAPI will indicate "UNABLE RTA", alerting the flight crew that a modification to the MOD route is required to achieve all of its constraints. In both the conflict and "UNABLE RTA" cases, the flight crew has several choices for dealing with the MOD route:

- Use AOP's MOD Route automatic resolution capability (see below) to adjust the current MOD Route until it is both conflict-free and meets all of its constraints, if possible, and then implement the MOD route.

- Modify the MOD route through manual inputs in the MCDU until the FMS MOD Route probe says the route is CLEAR and then implement the MOD route.

- Erase the MOD route.

- Implement the MOD route without modification.

The final option is available, since AOP does not prevent the flight crew from implementing a conflicted MOD route. Selecting this option, however, may be against operational procedures. The FMS MOD Route probe's function is merely to alert the crew to the impact of implementing the MOD route; the crew always make the final decision on any guidance change. 


\section{B. Tactical Planning Probe}

AOP's Tactical Planning probe supports the flight crew in safely making changes to certain tactical (non-LNAV and non-VNAV) guidance modes. If the change in guidance mode (see next section) is conflict-free, the SNAPI will indicate "CLEAR" on the MCP PLN line. If the change would result in a conflict, the SNAPI will indicate "CAUTION" or "NOT AVBL" on this line.

The Tactical Planning probe is triggered when it identifies "non-active displacements" in the track/heading and/or altitude windows on the MCP. Laterally, if the ownship is in LNAV, track hold (TRK HLD), or heading hold (HDG HLD) mode, any difference between the MCP track/heading window value and the aircraft's current track/heading value is considered a non-active displacement since this difference does not cause the aircraft's current guidance mode to attempt to achieve the MCP track/heading window value. Vertically, if the ownship is in VNAV or ALT HLD mode, any difference between the MCP altitude window value and the aircraft's current altitude is considered a non-active displacement. Either a lateral or vertical non-active displacement is sufficient to trigger AOP's Tactical Planning probe.

When triggered, the Tactical Planning probe alerts the flight crew to any conflicts that would exist if the crew were to simultaneously make all non-active displacements (both lateral and vertical) "active". This means assuming a switch to a guidance mode that would attempt to achieve the value in the MCP track/heading and/or altitude window. For lateral non-active displacements, the guidance mode assumed by the Tactical Planning probe depends on whether the MCP indicator for the track/heading window is set to TRACK or HEADING. If it is set to TRACK, the lateral mode is assumed to switch to TRK SEL. If set to HEADING, the lateral mode is assumed to switch to HDG SEL. For all vertical non-active displacements, the Tactical Planning probe assumes a switch to FLCH. No guidance mode switch is assumed for either lateral or vertical maneuvers if there is no non-active displacement.

In practice, the flight crew uses the Tactical Planning probe to determine whether a change to a tactical (nonFMS) guidance mode is safe to perform before executing the maneuver. For example, assuming that the MCP track/heading indicator is currently set to TRACK and the aircraft is in LNAV and VNAV, the pilot can simply adjust the MCP track window value as desired and the Tactical Planning will alert the flight crew to any conflicts that would occur if the lateral guidance mode was changed to TRK SEL (which initiates the turn) while the vertical guidance mode remains in VNAV. Alternatively, if the pilot had dialed in just a new MCP altitude value, the Tactical Planning will alert the flight crew to any conflicts that would occur if the vertical guidance mode was changed to FLCH (which initiates the maneuver) while the lateral guidance mode remains in LNAV. If both a track and altitude value were dialed in, the Tactical Planning will alert the flight crew to any conflicts that would occur if the lateral guidance mode was changed to TRK SEL and the vertical guidance mode was changed to FLCH, resulting in simultaneous lateral and vertical maneuvers (e.g., a climbing turn). If the Tactical Planning probe says CLEAR, the pilot can safely engage the desired tactical mode to achieve the new MCP window value.

\section{Active Route Recouple Probe}

AOP assumes that the active route in the FMS represents the most strategic plan of the flight crew and that, if the aircraft is not currently in LNAV and VNAV and following the active route, the objective of the flight crew is to eventually get back to this guidance state. In situations where the aircraft is not currently in LNAV and/or VNAV, but its position and altitude are such that these modes can be engaged (not just armed), the flight crew needs to assess whether engaging LNAV and VNAV will cause any undesirable impacts on AFR procedures. This is the purpose of the Active Route Recouple probe.

Similar to the MOD Route probe, AOP's Active Route Recouple probe has two main functions:

- Perform conflict detection on the active route to identify what conflicts would exist if LNAV and VNAV were both engaged at the current time.

- Determine if the active route would fail to achieve any RTA constraints that exist in the route if LNAV and VNAV were both engaged at the current time.

If the active route is conflict-free and meets all of its RTA constraints, the SNAPI will indicate "CLEAR" on the line entitled LNAV VNAV, alerting the flight crew that it is safe to engage LNAV and VNAV. If a conflict is detected along the active route, the SNAPI will indicate "CAUTION" or "NOT AVBL", alerting the flight crew that a conflict will be created if LNAV and VNAV are engaged. If the active route is conflict-free but unable to achieve an RTA constraint defined in the route, the SNAPI will indicate "UNABLE RTA", alerting the flight crew that a modification to the active route is required to achieve all of its constraints. In both the "CONFLICT" and "UNABLE RTA" cases, the flight crew has several choices:

- Use AOP's Active Route Recouple automatic resolution capability (see below) to adjust the active route until it is both conflict-free and meets all of its constraints, if possible, and then engage LNAV and VNAV. 
- Create a MOD route through manual inputs in the MCDU and modify it until the FMS MOD Route probe says the MOD route is CLEAR, then implement the MOD route.

- Engage LNAV and VNAV without modification to the active route.

As previously noted in the case of a conflicted MOD route, engaging LNAV and VNAV without modifying the active route to remove conflicts is possible but may be against operational procedures.

If the aircraft position and altitude are such that LNAV and VNAV cannot be engaged, the SNAPI will indicate "NO PROBE", indicating to the flight crew that attempting to select LNAV and VNAV at that time is an unprotected action.

\section{Maneuver Restriction (MR) Bands}

Similar to the function of the Tactical Planning probe, MR Bands are created to protect the flight crew when making tactical guidance changes. Unlike the Tactical Planning probe, MR Bands protect a wide range of lateral and vertical setting changes, but they do not guarantee that the resulting change will be completely conflict-free. Under autonomous flight rules, it is unacceptable for an AFR aircraft to make a trajectory change that results in a near-term conflict for either the ownship or the traffic aircraft. Although still a subject of research, a typical time horizon defining a near-term conflict is five minutes. MR Bands are designed to ensure that the tactical maneuver performed will not create a near-term conflict, though it purposefully does not protect creating a conflict with a larger time to first LOS, which may be operationally necessary in certain complex traffic situations. As such, MR Bands are primarily used by the flight crew when quick, safe maneuvers are required. The other conflict prevention capabilities, which ensure that the resultant change is completely conflict-free, are typically preferred when more planning time is available. In practice, MR Bands are often used to make fast decisions about the direction of tactical maneuvering (e.g., turning left or right) and then the Tactical Planning probe is used to refine the final MCP window value to achieve a completely conflict-free solution.

There are three types of MR Bands created by AOP:

- Track/Heading bands,

- Vertical Speed bands, and

- $\quad$ FLCH Altitude bands.

For Track/Heading bands, a range of track or heading values (depending on the MCP track/heading indicator) are probed, each assuming a lateral guidance mode of either TRK SEL or HDG SEL (again, based on the MCP track/heading indicator) and a vertical mode set to the aircraft's current vertical mode. The probe is purposefully limited to the longest look-ahead at which a near-term conflict could be detected for either the ownship or traffic aircraft. For each track/heading value probed that detects a conflict, that value is considered blocked. Adjacent values that are all blocked create a band stretching from the first contiguous track/heading value blocked to the last. The MR Band capability checks all track/heading values from +90 degrees to -90 degrees of the aircraft's current track/heading value, identifying all bands in this range. The bands are displayed on the PFD (see Fig. 5) and the Navigation Display (see Fig. 2).

Vertical Speed bands are calculated in a similar way. The vertical guidance mode is assumed to be V/R HLD, the lateral guidance mode is the aircraft's current

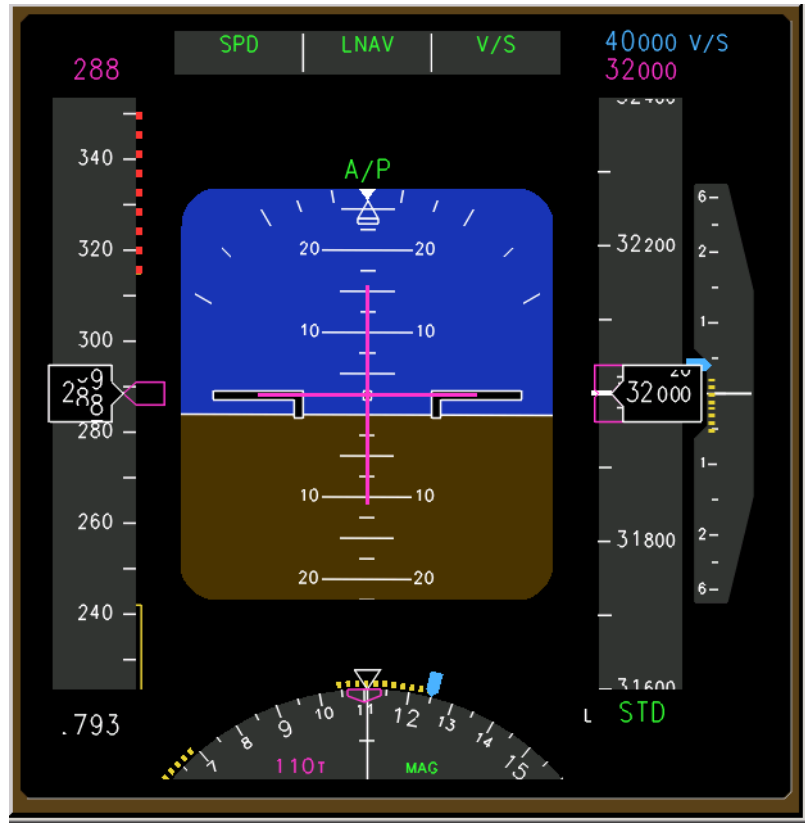

Figure 5. Displays of Track/Heading and Vertical Speed MR Bands on PFD lateral guidance mode, and a range of vertical rate values from $-8000 \mathrm{fpm}$ to $6000 \mathrm{fpm}$ are probed. The Vertical Speed bands created are displayed on the vertical speed tape of the PFD when the aircraft's vertical guidance mode is V/R HLD.

The crew use Track/Heading and Vertical Speed bands in similar manners. To ensure no near-term conflicts will arise, the pilot dials the appropriate MCP window value until it is a value that is in a gap between any displayed bands. The pilot then executes the maneuver by engaging the proper guidance mode (TRK/HDG SEL or V/R HLD). 
Since MR Bands are always being calculated, this approach works even when the aircraft is already in TRK/HDG SEL or V/R HLD, which is another difference between this capability and the Tactical Planning probe.

The FLCH altitude bands probe two cardinal altitudes $(+1000 \mathrm{ft}$ and $+2000 \mathrm{ft})$ above and two cardinal altitudes $(-1000 \mathrm{ft}$ and $-2000 \mathrm{ft})$ below the aircraft's current altitude. The vertical guidance mode is assumed to be FLCH and the lateral guidance mode is the aircraft's current lateral guidance mode. The FLCH altitude bands are displayed on the SNAPI (see Fig. 4). If a probed altitude value detects a near-term conflict, the SNAPI displays "BLOCKED" for that altitude, otherwise it displays no information on that altitude line.

The continuous availability of MR bands and the simple interface for entering values and executing new guidance modes (if any) makes the use of MR bands very effective for quick, evasive maneuvering.

\section{Strategic Intent-Based Conflict Resolution}

Strategic intent-based conflict resolution (SICR) is a capability within AOP that can find routes that the ownship could follow in full LNAV and VNAV guidance modes without conflict.

\section{A. Resolving Primary Conflicts}

The first and most essential role of SICR in the AOP is to resolve conflicts with the ownship's strategic intent, that is, when the ownship's intent is to follow the FMS active route in full LNAV and VNAV guidance modes. If the ownship is flying in this mode and the primary probe detects a conflict, SICR proposes a resolution route and enables the crew to execute this route as the new active route of the FMS. The resolution route is calculated to be conflict-free, that is, it resolves all conflicts with the current active route and creates no new conflicts.

Figure 6 illustrates an example of the procedure for resolving a primary conflict. In this example, SICR has generated three resolution routes (lateral, vertical, and combined), among which the vertical resolution is currently the "selected" resolution. The figure shows a page of the MCDU that is part of the interface to the AOP. The crew can upload this as a new FMS MOD Route by pressing the UPLOAD button on this MCDU page.

\section{B. Meeting a Required Time of Arrival}

When the active route includes an RTA, any SICR performed on the primary probe attempts to find a resolution route that meets the RTA and avoids all conflicts. When no such route is found, the SICR algorithm falls back to a "relaxed" resolution route that is conflict-free and misses the RTA by the minimum possible time difference.

The SICR capability can also be invoked when the current active route is already conflict-free but has missed an RTA. In that case, the resolution route (if one is found) will meet the RTA without creating any conflicts. For example, if an RTA is assigned that will require a delay maneuver, SICR is useful in plotting a conflict-free path that will absorb the required delay.

\section{Separation and Lookahead Requirements for SICR}

The assertion that the resolution route is conflict-free is based on a full "SICR probe" of an ownship trajectory based on the resolution route. The SICR probe is similar to the primary probe, but with two important differences:

- The SICR probe uses the resolution route in place of the current active route of the FMS.

- The separation requirements for the SICR probe are typically somewhat greater than the requirements for the primary probe.

The difference in separation requirements serves as a "buffer" to protect against minor differences that might

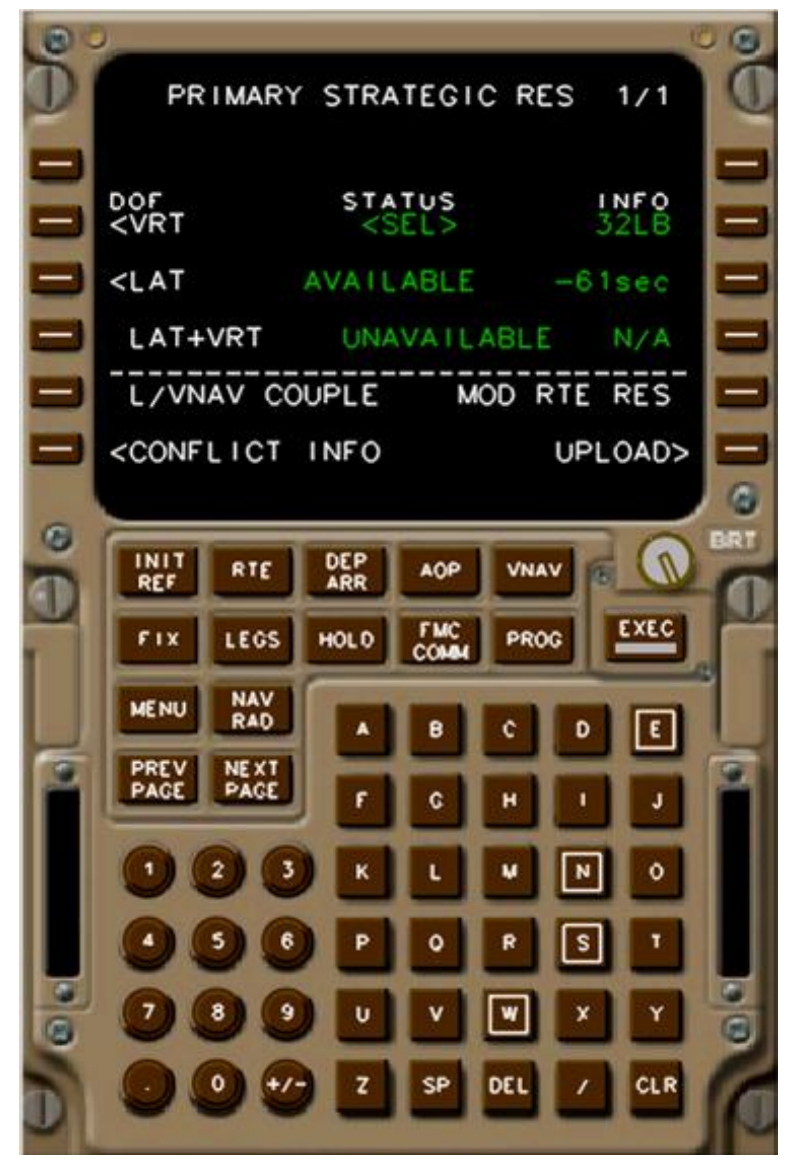

Figure 6. MCDU ready to upload a resolution route to the FMS. 
occur between the trajectory computed for the resolution route during SICR and the trajectory computed for the same route after it is executed as a new active route. Such differences could occur due to sensor error or other minor discrepancies between the reported ownship or traffic states from which the first trajectory is computed and the later states that are used when the second trajectory is computed.

A typical lookahead time for the SICR probe used in AOP research is 20 minutes, much longer than the 10 minutes typically used for the primary probe. The purpose of this difference is to avoid frequent repetition of the SICR procedure, and thereby to reduce crew workload. If SICR can find a resolution route that goes at least 20 minutes without conflict, and if there are no further changes to ownship or traffic intent (other than executing the resolution route) or to area hazards, and if the errors in the predictions on which SICR was based stay within the limits of their respective TPUBs, then at least 10 minutes should elapse from the beginning of this latest invocation of the SICR procedure until the next primary conflict is detected and needs to be resolved. Even when these conditions do not hold, it may be expected that conflicts will tend not to occur as frequently as they might if the lookahead for SICR were the same as for the primary probe.

\section{Resolving Conflicts with Other Probes}

In addition to the primary probe, SICR can resolve conflicts that are detected by the FMS MOD Route probe or by the Active Route Recouple probe. The crew can upload (and, if appropriate, execute) the resulting resolution route to obtain a new, conflict-free FMS MOD route or active route according to the procedures described in Section VII ("Conflict Prevention").

The SICR algorithms for these two probes are essentially the same as for the primary probe. The objective is to modify the given route (MOD route or active route) so that it is conflict-free.

\section{E. SICR Algorithms}

The AOP is designed and architected to provide a choice among different algorithms for SICR. The current implementation uses the pattern-based genetic algorithm (PBGA), ${ }^{9,10}$ configurable for lateral, vertical, and/or combination maneuvers. A resolution route generated by PBGA is the result of applying a parameterized transformation (a maneuver pattern) to an input route, usually initiating a "maneuver" away from that route at one point and returning to it at another point. For example, in order to resolve a primary conflict, PBGA will apply a maneuver pattern to the current active route of the FMS. Figure 7 shows the form and parameters of the Direct Intercept pattern for lateral maneuvers, one of several maneuver patterns currently implemented in PBGA.

In order to converge toward a conflict-free route, PBGA includes a fitness function that assesses the amount of "conflicted" airspace (including possible new conflicts due to maneuvers) on each side of a route, and favors routes that are closer to the "edge" of this airspace. A conflict-free route is always preferred over one with conflicts. The fitness function penalizes a missed RTA.

Past versions of AOP supported a PBGA tuned to a flowcorridor operational concept involving self-separation called Dynamic Multi-track Airways ${ }^{11}$ as well as an early perturbation-based genetic algorithm for the AFR concept. ${ }^{12}$

\section{F. Optimization of Resolution Routes}

Among the routes generated by PBGA that are conflictfree (and meet their RTAs when applicable), the fitness function of PBGA favors more "optimal" routes. The AOP can be configured to use any of several different optimization

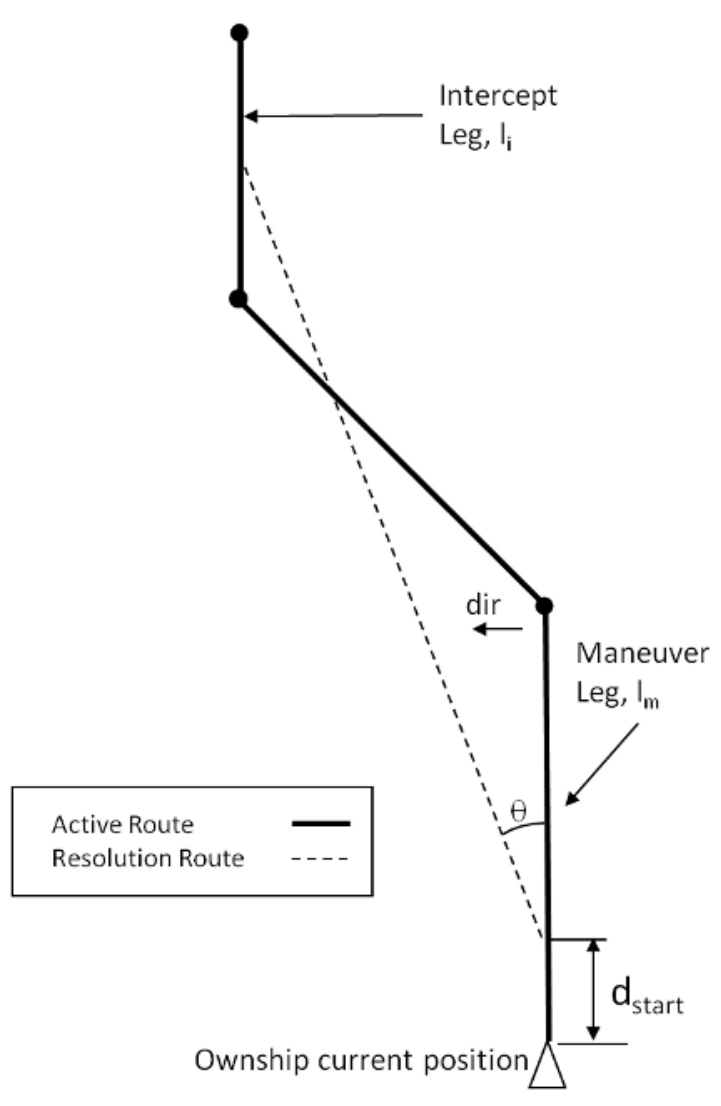

Figure 7. An example of a maneuver pattern in PBGA: the Direct Intercept pattern.

metrics for these route, for example, pattern type, shape, and size; time to reach the destination; or fuel remaining at the destination. 


\section{G. Strategic Reroute}

The Strategic Reroute capability in the AOP enables the crew to request a conflict-free route that will "reconnect" to the existing active route when the ownship has left the active route. To accomplish this, the AOP invokes SICR using the current active route as input to PBGA, which applies a pattern especially designed for this purpose. This "reconnect" pattern bases the start of its maneuver on the ownship's current state rather than on the active route, but ends the maneuver on the active route.

\section{H. Automatic Initiation of Conflict Resolution}

In order to minimize the perceived time to perform the $\mathrm{CR}$ algorithm, the AOP will automatically initiate strategic resolution (if available) upon first detection of the conflict. If the MCDU is currently displaying one of the pages corresponding to any of the SICR capabilities (primary CR, FMS MOD route CR, FMS active route recouple $\mathrm{CR}$, or strategic reroute), the AOP will initiate the corresponding strategic resolution when applicable. Otherwise, the selection of the SICR function to perform is based on the history of pages displayed on the MCDU and on an underlying prioritization of the SICR functions.

\section{Tactical Intent-Based Conflict Resolution}

The Tactical Intent-based Conflict Resolution (TICR) function of AOP enables it to provide advisories to ensure self-separation when any tactical guidance mode ${ }^{\dagger \dagger \dagger}$ of the aircraft is engaged or as a fallback from SICR in the event that SICR is unable to provide a resolution. ${ }^{13}$ The capabilities of TICR include exhaustive probing of the solution space, conflict-avoidance relaxation, weather-conflict relaxation, maneuver stabilization, and implicit coordination, as explained below. In support of these capabilities, TICR uses fitness rules to weigh these parameters in a manner that allows TICR to converge on the best possible conflict-free manuever in any given situation. Where constraint or conflict relaxation is allowed, a fitness penalty is added to place these less desirable solutions in the proper relationship with conflict-free solutions.

\section{A. Tactical Intent-Based Maneuvers}

Any advisory (or tactical resolution) provided by TICR is a maneuver defined by a tactical guidance mode and (in most cases) by a heading, track angle, or altitude. Lateral maneuvers can be defined by the HDG SEL or TRK SEL modes, in which case the maneuver is a turn to capture a heading or track angle. Vertical maneuvers can be defined by a FLCH or ALT HLD mode; a FLCH maneuver would climb or descend to capture an altitude. The crew can implement the advisory (if they deem it acceptable) by setting the advised heading, track angle, or altitude in the track/heading or altitude window of the MCP and then engaging the advised guidance mode.

\section{B. Exhaustive Search of Solution Space}

The TICR function performs "sweeps," each of which probes a discrete set of manuevers consisting of a single guidance mode and advised headings, track angles, or altitudes spaced at regular intervals, usually 1 degree (lateral) or 1000 feet (vertical) apart, within a predefined "sweep" range. This range is currently defined to be $+/-60$ degrees from the current track or heading and $+/-10,000$ feet from the current altitude, whichever applies. Experience has shown that when these maneuvers are probed in sequence, the first conflict-free maneuver found is not always the most desirable, even if the sequence of probes is carefully constructed. Therefore, each "sweep" probes its entire solution space, allowing the algorithm to evaluate each maneuver according to the relevant fitness rules, and selects the maneuver whose fitness was best.

\section{TICR as a Strategic Separation Assurance Fallback}

The SICR capability of the AOP is a powerful tool for resolving conflicts and keeping the aircraft flying in a strategic mode with full FMS guidance. ${ }^{14}$ Certain conditions, however, such as local airspace complexity or conflict proximity, may prevent SICR from providing a suitable conflict-free advisory. In this situation, the TICR capability will automatically activate and will provide a conflict-free advisory if it is able, ensuring continuity of separation assurance. The advisory will recommend leaving FMS guidance and entering a tactical guidance mode. The Strategic Reroute capability of the AOP can be used later to return safely to full FMS guidance. The AOP will also allow the pilot to force TICR guidance to be provided in lieu of SICR capability, if desired for an operational reason.

\footnotetext{
${ }^{\dagger \dagger}$ As defined earlier, a tactical guidance mode is any mode other than LNAV and VNAV.
} 


\section{Maneuver Stability}

Where a range of conflict-free maneuvers is available, the TICR algorithm will weigh these maneuvers with respect to their their impact on the ownship's current situation. For example, if the ownship is currently turning left and conflict-free maneuvers exist both to the left and to the right, the fitness function of TICR will give maneuvers to the left a higher preference than maneuvers to the right, since that does not require a change in direction of turn. Similar considerations hold for altitude maneuvers. Additionally, a maneuver that requires a small change in heading, track angle, or altitude will be favored over a maneuver requiring a larger change. Where a range of conflict-free maneuvers lies between conflicted maneuvers, the tactical resolution will be biased toward the center of that range. This minimizes the chance that a minor change in the predictions on which the conflicts were based will cause a new conflict and require the ownship to maneuver again.

\section{E. Implicit Coordination}

Another factor influencing the fitness of conflict-free maneuvers is the desire to coordinate maneuvers of the ownship and traffic aircraft. Two aircraft flying under AFR are allowed to maneuver simultaneously; from the perspective of one of those aircraft, these simultaneous maneuvers include an "ownship" maneuver and a "traffic" maneuver. A "sweep" performed by TICR compares each of its ownship trajectories against the existing traffic trajectories, not against trajectories that would result from a traffic maneuver. Coordination of an ownship maneuver occurring simultaneously with a traffic aircraft maneuver ensures that the new ownship trajectory is not in conflict with the new traffic trajectory after both maneuvers are performed. This allows the conflict between the two aircraft to be resolved as efficiently as possible and without the risk of "sidewalk" behavior, in which simultaneous maneuvers by the two aircraft repeatedly put them in conflict with each other. ${ }^{\text {tot }}$ Explicit coordination would require a (possibly lengthy) sequence of communications between the aircraft in order to establish agreement. The algorithm used by TICR, developed by the Formal Methods group at NASA Langley, performs implicit coordination, in which a "coordinated" maneuver by one aircraft is guaranteed not to conflict with any possible "coordinated" maneuver of the other aircraft. A coordinated resolution will be given preference over a non-coordinated resolution.

Only one-on-one conflicts can be coordinated by the existing algorithm. Maneuvers to avoid simultaneous conflicts with more than two aircraft cannot be coordinated and this metric is not considered in their overall fitness.

\section{F. Conflict-Avoidance Relaxation}

A conflict-free solution is always preferred, but it is not possible to find a completely conflict-free solution in all situations. While an aircraft flying under AFR is not permitted to maneuver in any way that creates a near-term conflict, the AOP may advise a conflict resolution that improves upon the current situation by suggesting a maneuver that avoids all near-term conflicts but creates one or more far-term conflicts. In these situations, when the ownship executes such a maneuver, all aircraft involved are given more time to find alternate solutions.

\section{G. Weather-Conflict Relaxation}

While avoiding LOS with a traffic aircraft is mandatory, the same does not always apply to weather. An experiment focused on integrated traffic-weather avoidance ${ }^{15}$ evaluated the ability of the AOP to deal with weather when the information about weather is limited to the capabilities of the on-board radar system. Assuming no ability to predict weather movement in that case, weather cells tend to "pop up" on the path of the aircraft just as they come into radar range, usually with a first LOS that is much sooner than the lookahead of the primary probe. To address these issues, each "core" weather cell, represented by a polygonal region of the airspace, is surrounded by a larger "avoidance zone," which is a configurable convex buffer around the core weather cell. AOP allows no resolution to penetrate a core weather cell. The TICR algorithm will also attempt to not penetrate any avoidance zone, but if it finds no such resolution, it will allow a resolution that enters an avoidance zone. The fitness function is biased to minimize penetration and to penalize maneuvers that approach too close to the core hazard.

\section{Crew Workload and Coordination of Efforts}

With the many trajectories AOP probes, and the various conflict and resolution alerts available to the pilot, maintaining acceptable crew workload has been a continual focus of the AOP display and algorithm development. To this end, two key concepts, alert levels and priority rules, have been employed to present the flight crew with

\footnotetext{
Nhe term "sidewalk" alludes to a situation in which two pedestrians approach each other along a sidewalk. Each makes a slight change in direction — one to his left, the other to her right - in an attempt to step out of the other's way. In extreme cases, after a series of well-meaning but unfortunate decisions of this sort, the two stop face to face.
} 
clear and concise information, thus reducing the necessary workload and allowing for improved real-time collaboration across aircraft. The combination of these two capabilities directly addresses the issues of when conflict information is communicated to the flight crew and how it is to be presented. By filtering conflicts according to their AFR alert levels, AOP reduces the amount of information that must be displayed to the flight crew. AOP defines the "priority" of aircraft in conflict (that is, which one has the "right of way"), and initially alerts the conflict to the crew only when the ownship has lower priority than the traffic aircraft, thereby helping to avoid uncoordinated responses to conflicts. Like implicit coordination, this addresses the "sidewalk" scenario.

The following subsections provide greater detail about alert levels and priority rules.

\section{A. Alert Levels}

A key concept underlying the AOP design is to provide dependable conflict alerting to the pilot in all flight modes, in order to support the flight crew's use of all guidance options available on the flight deck. ${ }^{16}$ In order to provide such alerting in a concise and clear manner, alert levels are assigned to each conflict for later use in prioritization and filtering. The rule set containing the logic for assigning these levels is defined in an alerting scheme, which may be tailored to the experiment at hand. Aspects such as hazards type (traffic aircraft or area hazard such as SUA), trajectory type, and time to separation loss are typically key factors in determining the precise alert level of a given conflict. The displays then use alert levels to determine which conflicts to display and to provide visual queues to the flight crew as to the nature of each conflict. Currently, AOP supports two displayed alert levels and one non-displayed alert level.

\section{B. Priority Rules}

Priority rules help avoid uncoordinated maneuvers (and "sidewalk" behavior) by determining which aircraft in a conflict pair has the "right of way." When the conflict's time to first LOS is close to the full lookahead time for the conflict probe, the "lower priority" aircraft is alerted to the conflict first, allowing that aircraft to execute a maneuver to resolve the conflict. Should the time to first LOS fall below a specific threshold, the "priority" aircraft will also be alerted to the conflict

As with the alerting levels, priority rules are applied equally under all flight modes in order to support the full range of automation on the flight deck. Aircraft flying under IFR are always given the right of way over AFR aircraft, relying on the ability of the AFR aircraft to resolve the conflict without intervention from a ground controller. For conflicts between autonomous aircraft, the algorithm assigns priority according to a range of other characteristics of the aircraft; for example, all other things being equal, an aircraft flying level has priority over a climbing aircraft.

\section{State-Based Conflict Detection and Resolution}

Complementing the intent-based algorithms described above, AOP employs a state-based conflict detection and resolution $(C D \& R)$ capability to handle extremely short term conflicts and losses of separation with traffic aircraft. Using aircraft position and velocity vector information for both ownship and traffic aircraft, the state-based algorithm uses simple state projections to predict and resolve near-term traffic conflicts. Although these projections ignore all information regarding the future intent of both aircraft, such projections are acceptable for the short time horizons encountered when tactical maneuvers are required in close proximity to loss. These projections are probed for conflicts using configurable separation standards that are independent of those used by the intent-based probes. State-based conflicts, if detected, are reported in a manner consistent with their intent-based counterparts, while state-based resolutions are advised as specific tactical guidance settings similar to TICR. State-based versions of MR bands are also generated as part of the state-based advisories and are displayed in the same manner as the intentbased MR bands.

The state-based CD\&R approach within AOP has evolved over time from an intent-based override capability, where a detected state-based conflict would automatically disengage the intent-based CD\&R capabilities at medium times to LOS (3-5 minutes), to a separation loss recovery fallback following strategic and tactical intent-based detection and resolution failures. The former approach was employed as part of a simulation conducted in $2004 .{ }^{17}$ As a result of this experiment, several issues were brought to light. The most significant was the tendency for the state-based system to incorrectly override the intent-based system during initial descents to the terminal airspace. This behavior was caused by the inaccuracy of the state-based projection of constant groundspeed and vertical rate during the descent phase of flight.

Recently, AOP's previous approaches to state-based CD\&R $\left(\mathrm{NLR}^{18}\right.$ and $\left.K B 3 \mathrm{D}^{19}\right)$ have been replaced with Airborne Coordinated Conflict Resolution and Detection (ACCoRD) algorithms developed at NASA Langley 
Research Center (LaRC). ${ }^{20}$ Integrated into AOP to support separation loss recovery, this approach becomes active shortly before loss (typically 30 seconds or less) in order to assist the flight crew in effectively managing, or possibly avoiding, the impending protected zone penetration. It should be noted that the new triggering mechanism used for initiation of state-based CD\&R is a conflict detected by the intent-based conflict detection capability whose time to first LOS falls below a prescribed state-based activation threshold.

Once active, the state-based conflict detector probes for conflicts using a look-ahead time of five minutes. Should one or more state-based conflicts be detected, the conflict with the shortest time to first LOS will be reported by AOP; this is consistent with the resolution approach of the ACCoRD algorithm, which gives special attention to the most critical conflict. In addition to conflict first and last LOS positions and time, the resolution function provides independent guidance targets for track and vertical rate to optimally avoid conflicts with all traffic, not just the most critical one, thereby offering a clear path away from the conflict without introducing additional conflicts and subsequent maneuvers. When possible, the targets are selected by an algorithm that implicitly coordinates the ownship maneuver with the possible maneuvers of the traffic aircraft causing the most critical conflict. ${ }^{21}$ Like the implicit coordination algorithm in TICR, this algorithm helps to avoid "sidewalk" behavior. In addition, the resolution function indicates whether the guidance targets are implicitly coordinated, whether the track target avoids the protected zone, and whether the track target or the vertical rate target provides a better resolution. In the event that no state-based conflict is detected (for example, when the relevant intent-based conflict is caused by a turn), a state resolution indicating that the aircraft maintain its present track and vertical rate is provided to the flight crew.

\section{Advances in the Integration of Flight-Deck Functions}

As self-separation research has progressed at NASA LaRC, new AOP capabilities have been developed and existing capabilities extended to meet the challenges of an advanced, distributed control operational concept. This has led to new and novel approaches within several areas of separation assurance research, including some that extend beyond the specific application to the flight deck. Some of the highlights are presented below.

\section{A. Integration of Strategic and Tactical CD\&R.}

To support the many complex guidance modes available to the flight crew, AOP has a fully integrated, adaptive suite of CD and CR functions. The primary focus is on intent-based conflict detection with either a strategic (SICR) or tactical (TICR) resolution capability, depending on the current aircraft guidance mode. In challenging conflict scenarios, if strategic resolutions become unavailable, AOP automatically falls back to TICR; once tactical maneuvering is complete, AOP's Strategic Reroute function helps the flight crew reconnect the aircraft position to the current FMS active route. In the rare cases where a loss of separation does occur, AOP automatically engages state-based conflict detection and resolution capabilities to support the flight crew in safely regaining separation. Once separation has been reestablished, intent-based conflict detection and resolution automatically reengage. AOP's approach to CD\&R anticipates the needs of the flight crew and provides smooth transitions in and out of strategic flight, as necessary.

\section{B. Use of Conflict Prevention Capabilities.}

AOP supports the flight crew in making safe changes to the aircraft's guidance settings that are not driven by separation assurance needs. By determining the impacts of a proposed guidance change prior to execution, AOP enables the flight crew to maintain self-separation when making any guidance changes within the wide range of potential FMS-based and non-FMS-based guidance settings available to the flight crew. In the case of FMS-based guidance, if an FMS MOD route would result in a conflict or an unmet RTA constraint, AOP provides automated resolution capabilities to enable the flight crew to adjust the MOD route to meet the requirements of airborne selfseparation while still achieving the objectives of their original proposed change. In support of non-FMS-based guidance, AOP monitors non-active changes to the MCP track/heading and altitude window values, enabling the flight crew to probe tactical maneuvers quickly and effectively before switching to a mode that will capture the new window value.

\section{Support for distributed control.}

The main challenge for any distributed control ATC environment is how to make sure that simultaneous maneuvers between aircraft do not result in undesired conflicts or losses of separation. AOP's intent-based (SICR and TICR) and state-based CD\&R capabilities have been tuned to the needs of a distributed control environment. AOP's use of priority rules reduces the chance that two aircraft will simultaneously maneuver to resolve the same conflict by giving the non-priority aircraft a chance to resolve the conflict before it is alerted to the priority aircraft. 
When both aircraft do maneuver to resolve the same conflict, AOP's tactical and state-based resolutions use implicit coordination rules, reducing the chance that the resolutions selected by each aircraft will result in a new conflict.

\section{Advanced Trajectory Prediction for All Aircraft Guidance Modes.}

Unlike the trajectory predictor within the FMS, AOP's trajectory prediction algorithms can predict all of the possible guidance mode combinations available to the flight crew, including fully FMS-based modes (LNAV/VNAV), non-FMS-based modes, and hybrid combinations of FMS-based and non-FMS-based modes (e.g., LNAV and FLCH). AOP is also capable of predicting conditional switches in guidance modes. For example, an aircraft in TRK HLD with LNAV in the armed state will switch to LNAV only if the aircraft's position gets within a defined distance of the FMS active route. The development of AOP's algorithms to predict conditional guidance mode switching required capabilities beyond the state-of-the-art in trajectory prediction at the time and led directly to advances in the conceptual modeling of trajectory predictors. ${ }^{22}$

\section{E. Fast Conflict Detection Algorithms with Built-In Uncertainty Handling.}

Several advances were made to AOP's conflict detection algorithms to deal with the challenges of the airborne self-separation requirement. To meet the need for completing CD for several hundred aircraft in a few hundred milliseconds, AOP algorithms forgo time consuming uniform-trajectory-discretization and pre-filtering techniques for a highly efficient and more accurate continuous-motion technique. To handle the variation in trajectory prediction accuracy between different trajectory generation sources (ownship vs. traffic), the TPUBs approach to uncertainty bounds was developed and integrated directly into both the trajectory generation and conflict detection algorithms. The end result is a fast, accurate $\mathrm{CD}$ algorithm with a highly customizable approach to handling prediction uncertainty.

\section{F. Handling Mixed Traffic Intent Environments.}

AOP's approach to handling traffic aircraft data has been developed to accept a wide range of potential data sources, including ADS-B and SWIM. For ADS-B, AOP supports the full range of potential future ADS-B data available, including both target state and multiple-TCP trajectory change reports. For each traffic aircraft, AOP identifies the most strategic source of ADS-B data available that passes a set of real-time consistency checks with respect to the aircraft's current state vector report and builds a trajectory based on that data. Recently, AOP has added processing to integrate uplinked SWIM data into this approach, assuming SWIM is more strategic than ADSB target state data, but less desirable than ADS-B trajectory change report data being sent directly from the traffic aircraft.

\section{G. Integrated Conflict Resolution and Flight Optimization.}

AOP's SICR capability has been designed to provide routes for upload into the FMS that are not only conflictfree but also have been optimized based on a predefined objective by the flight crew. Options include optimizing global trajectory parameters (e.g., fuel burned, time or distance flown) or optimizing based on the geometry of the specific pattern used (e.g., minimize offset distance). Unlike functions dedicated solely to route optimization, SICR optimizes routes while giving priority to resolving and avoiding conflicts. In particular, SICR optimizes routes only after a conflict-free solution has been found. This approach may sacrifice some level of flight optimization, but increases the probability of finding conflict-free solutions, which is of primary importance for conflict resolution.

\section{H. Advanced Weather Avoidance.}

Unlike some CR approaches, each resolution generated by an AOP resolution algorithm (SICR and TICR) provides a single, integrated solution to resolve all detected traffic and area hazard (weather, SUA) conflicts. This means the resolutions provided to avoid both traffic and area hazards are no more complex than the solutions available for just traffic conflicts. TICR adds the ability to relax the need to avoid certain weather hazards (avoidance zones) if the only alternative is to remain in conflict with a traffic aircraft. Both SICR and TICR algorithms continue to be enhanced to work with various sources of weather data, including both on-board weather radar data and uplinked weather information. 


\section{Conclusion}

The architecture, algorithms, and implementation of AOP provide powerful tools to support AFR operations across a wide range of conditions. The trajectory prediction and conflict detection functions in AOP use a broad selection of data available from avionics in order to make useful predictions of all aircraft trajectories, supporting a comprehensive range of predictable guidance modes of the ownship aircraft (including FMS-guided flight, guidance independent of the FMS, and partial tactical overrides of the FMS) and several levels of enhanced or degraded data describing traffic aircraft. The conflict detection algorithm and its controlling parameters can be precisely tuned to alert flight crews to developing problems when they should be solved, and to avoid (to the extent practical) false notifications of problems or excessive notifications of problems that are less urgent. Moreover, several possible pitfalls of self-separation, such as the so-called "sidewalk" behavior, have been identified and effectively eliminated by multiple, complementary layers of protection.

The maneuvers that AOP's conflict resolution algorithms generate in response to conflicts and other problems are simple, efficient (due to well-integrated optimization criteria) and robust. Most resolutions are "global," simultaneously meeting constraints such as required time of arrival (when applicable) and accounting for all known hazards, including hazards of several disparate types (such as aircraft, SUAs, and weather cells). These and other functions supporting flight crews adapt automatically to changing conditions either in the airspace or in the ownship's guidance.

The history of AOP shows a proven track record of usefulness in the investigation of a variety of aspects of the AFR concept within a variety of detailed definitions of the concept. The capabilities developed for AOP represent significant advances in the state of the art. New capabilities continue to be integrated into the suite and refined. It is expected that ongoing and future research efforts will continue to use versions of this prototype system to investigate and improve the AFR concept.

\section{References}

${ }^{1}$ Wing, D. J. and Cotton, W. B., “Autonomous Flight Rules: A Concept for Self-Separation in U.S. Domestic Airspace," NASA TP-2011-217174, November 2011.

${ }^{2}$ Finkelsztein, D., Lung, T., Vivona, R., Bunnell, J., Mielke, D., and Chung, W., "Airspace and Traffic Operations Simulation for Distributed ATM Research and Development," AIAA-2005-6488, AIAA Modeling and SimulationTechnologies Conference, AIAA, Washington, DC, 2005.

${ }^{3}$ Cotton, W. B., "New Directions in Air Traffic Control at Kennedy Airport," Master's Thesis, Course XVI, Massachusetts Institute of Technology, Cambridge, MA, 1965.

${ }^{4}$ Ballin, M. G., Sharma, V., Vivona, R. A., Johnson, E. J., and Ramiscal, E., “A Flight Deck Decision Support Tool for Autonomous Airborne Operations," AIAA-2002-4554, AIAA Guidance Navigation, and Control Conference, AIAA, Washington, DC, 2002.

${ }^{5}$ Wing, D. J. and Cotton, W. B., "For Spacious Skies: Self-Separation with 'Autonomous Flight Rules' in US Domestic Airspace," AIAA-2011-6865, AIAA Aviation Technology, Integration, and Operations Conference, AIAA, Washington, DC, 2011.

${ }^{6}$ Karr, D. A., Roscoe, D. A., and Vivona, R. A., "An Integrated Flight-Deck Decision-Support Tool in an Autonomous Flight Simulation," AIAA-2004-5261, AIAA Conference on Modeling and Simulation Technologies, AIAA, Washington, DC, 2004.

${ }^{7}$ Paielli, R. A. and Erzberger, H., "Conflict Probability Estimation for Free Flight," Journal of Guidance, Control, and Dynamics, Vol. 20, No. 3, May-June 1997, also published as NASA TM-110411.

${ }^{8}$ Karr, D. A. and Vivona, R. A., "Conflict Detection Using Variable Four-Dimensional Uncertainty Bounds to Control Missed Alerts," AIAA-2006-6057, AIAA Guidance, Navigation and Control Conference, AIAA, Washington, DC, 2006.

${ }^{9}$ Vivona, R. A., Karr, D. A., and Roscoe, D. A., "Pattern-Based Genetic Algorithm for Airborne Conflict Resolution," AIAA-2006-6060, AIAA Guidance, Navigation and Control Conference, AIAA, Washington, DC, 2006.

${ }^{10}$ Vivona, R. A., Karr, D. A., Roscoe, D. A., DePascale., S. M., and Consiglio, M., "Experimental Performance of a Genetic Algorithm for Airborne Strategic Conflict Resolution," AIAA-2009-5748, AIAA Guidance, Navigation and Control Conference, AIAA, Washington, DC, 2009.

${ }^{11}$ Wing, D. J., Smith, J. C., and Ballin, M. G., "Analysis of a Dynamic Multi-Track Airway Concept for Air Traffic Management," NASA TP-2008-215323, 2008.

${ }^{12}$ Mondoloni, S. and Conway, S., "An Airborne Conflict Resolution Approach Using a Genetic Algorithm," AIAA-20014054, AIAA Guidance, Navigation and Control Conference, AIAA, Washington, DC, 2001.

${ }^{13}$ Wing, D. J., Vivona, R. A., and Roscoe, D. A., "Airborne Tactical Intent-Based Conflict Resolution Capability", AIAA-2009-7020, AIAA Aviation Technology, Integration and Operations Conference, AIAA, Washington, DC, 2009.

${ }^{14}$ Consiglio, M., Hoadley, S., Wing, D., and Baxley, B., "Safety Performance of Airborne Separation: Preliminary Baseline Testing," AIAA-2007-7739, AIAA Aviation Technology, Integration and Operations Conference, AIAA, Washington, DC, 2007. 
${ }^{15}$ Consiglio, M., Chamberlain, J., and Wilson, S., "Integration of Weather Avoidance and Traffic Separation," IEEE/AIAA Digital Avionics Systems Conference, IEEE, 2011, pp. 3B4-1-3B4-14.

${ }^{16}$ Krishnamurthy, K. and Eischeid, T., "Alerting Logic and User Interfaces for Airborne Conflict Management in ATOL Software Build 2.0”, Version 3, August 27, 2003.

${ }^{17}$ Barhydt, R., Kopardekar, P., Battiste, V., Doble, N., Johnson, W., Lee, P., Prevot, T., and Smith, N. T., "Joint NASA Ames/Langley Experimental Evaluation of Integrated Air/Ground Operations for En Route Free Maneuvering," USA/Europe Seminar on Air Traffic Management Research and Development, Baltimore, MD, 2005.

${ }^{18}$ Hoekstra, J. M., "Designing for Safety the Free Flight Air Traffic Management Concept," NLR-TP-2001-313, National Aerospace Laboratory, Amsterdam, The Netherlands, 2001.

${ }^{19}$ Dowek, G., Muñoz, C., and Carreño, V., "Provably Safe Coordinated Strategy for Distributed Conflict Resolution," AIAA-2005-6047, AIAA Guidance Navigation, and Control Conference, AIAA, Washington, DC, 2005.

${ }^{20}$ Muñoz, C., Butler, R., Narkawicz, A., Maddalon, J., and Hagen, G., “A Criteria Standard for Conflict Resolution: A Vision for Guaranteeing the Safety of Self-Separation in NextGen," NASA TM-2010-216862, 2010.

${ }^{21}$ Narkawicz, A. and Muñoz, C., "State-Based Implicit Coordination and Applications," NASA TP-2011-217067, March 2011.

${ }^{22}$ Vivona, R.A., Cate, K.T., and Green, S.M.: "Abstraction Techniques for Capturing and Comparing Trajectory Predictor Capabilities and Requirements", AIAA-2008-7408, AIAA Conference on Guidance, Navigation, and Control, AIAA, Washington, DC, 2008 